\title{
A self-consistent leptonic-hadronic interpretation of the electromagnetic and neutrino emissions from blazar TXS 0506+056
}

\author{
Gang $\mathrm{CAO}^{1}$, Chuyuan $\mathrm{YANG}^{2}$, Jianping $\mathrm{YANG}^{3}$ and Jiancheng $\mathrm{WANG}^{2}$ \\ ${ }^{1}$ Department of Mathematics, Yunnan University of Finance and Economics, Kunming \\ 650221, Yunnan, P. R. China \\ ${ }^{2}$ Yunnan Observatories, Chinese Academy of Sciences, Kunming 650216, Yunnan, P. R. \\ China \\ ${ }^{3}$ Yunnan Agricultural University, Kunming 650201, Yunnan, P. R. China \\ *E-mail: gcao@ynufe.edu.cn \\ Received ; Accepted
}

\begin{abstract}
The potential association between the blazar TXS 0506+056 and the neutrino event IceCube$170922 \mathrm{~A}$ provides a unique opportunity to study the possible physical connection between the high-energy photons and neutrinos. We explore the correlated electromagnetic and neutrino emissions of blazar TXS $0506+056$ by a self-consistent leptonic-hadronic model, taking into account particle stochastic acceleration and all relevant radiative processes self-consistently. The electromagnetic and neutrino spectra of blazar TXS 0506+056 are reproduced by the proton synchrotron and hybrid leptonic-hadronic models based on the proton-photon interactions. It is found that the hybrid leptonic-hadronic model can be used to better explain the observed $\mathrm{X}$-ray and $\gamma$-ray spectra of blazar TXS 0506+056 than the proton synchrotron model. Moreover, the predicted neutrino spectrum of the hybrid leptonic-hadronic model is closer to the observed one compared to the proton synchrotron model. We suggest that the hybrid leptonic-hadronic model is more favored if the neutrino event IceCube-170922A is associated with the blazar TXS 0506+056.
\end{abstract}

Key words: BL Lacertae objects: individual( TXS 0506+056 ) - galaxies: active - galaxies: gamma-ray - neutrino - radiation mechanisms: non-thermal

\section{Introduction}

Neutrino observation by IceCube has opened up a new window in the study of nonthermal processes in astrophysical objects. However, the sources responsible for the neutrino emission have not been identified so far. As the neutrinos are not absorbed when interacting with the background photons or the matters, they can be detected even though the source is far away. The observed distribution of their arrival direction suggests a predominantly extragalactic origin. Extragalactic sources, such as active galactic nuclei (Murase et al. 2014; Petropoulou 2015; Padovani 2016; Gao et al. 2017; Murase et al. 2018; Gao et al.
2019), $\gamma$-ray burst (Murase et al. 2006; Petropoulou 2014) and supernova (Murase et al. 2011; Petropoulou 2017), have been proposed as the potential high-energy neutrino sources. Blazars are believed to be the most promising candidate sources with high-energy neutrino emission.

The IceCube observation recently reported the detection of a neutrino event( IceCube-170922A ), which is coincidence with the blazar TXS 0506+056 during its flaring state (IceCube Collaboration 2018a; IceCube Collaboration 2018b). Following the neutrino alert, the blazar TXS 0506+056 is detected in a multi-wavelength campaign, ranging from the ra-

(C) 2014. Astronomical Society of Japan. 
dio to $\gamma$-ray bands (IceCube Collaboration 2018b). The multiwavelength observations characterize the polarization, variability and energy spectrum of the blazar TXS 0506+056. This source is also first detected in very high-energy $\gamma$-ray bands with the MAGIC Cherenkov telescopes. A chance coincident of the high-energy neutrino with multi-wavelength flare is rejected at a $3.5 \sigma$ level. The potential association between the activity of TXS $0506+056$ and the neutrino event suggests that this object could be the counterpart of the neutrino event.

Blazars are a subclass of radio-loud active galactic nuclei powered by supermassive black holes. Their radiation is thought to originate in a relativistic jet oriented at a small angle with respect to the line of sight. Blazars are often classified into BL Lac objects and flat spectrum radio quasars (FSRQs). BL Lacs have weak or absent emission lines, while FSRQs usually show strong broad emission lines. The spectrum energy distributions (SEDs) of blazars are characterized by non-thermal continuum spectra with a broad low-energy component from radio-UV to X-ray and a broad high-energy component from $\mathrm{X}$-ray to $\gamma$-ray. It is generally accepted that the low-energy component of blazar SEDs is produced by synchrotron emission from relativistic electrons accelerated in the jet of blazar. The high-energy component is often interpreted as the inverse Compton (IC) upscattering of ambient soft photons by the accelerated electrons (e.g., Böttcher 2007). The soft photons can be either synchrotron photons within the jet( the synchrotron self-Compton, SSC, process, Maraschi et al. 1992; Bloom and Marscher 1996), or the photons external to the jet ( the External Compton, EC, process). These external photons may be the UV accretion disk photons(Dermer \& Schlickeiser 1993), the accretion disk photons reprocessed by broad-line region clouds (Sikora et al. 1994), or infrared photons from the dust torus (Blaźejowski et al. 2000). The above scenario is called the leptonic model. Such models have achieved great successes in explaining the multi-wavelength emission and variability from blazars (Böttcher 2002; Weidinger \& Spanier 2010).

It is physically plausible that the protons are co-accelerated with the electrons to very-high energy by the same mechanism in the jet of blazar. In the so-called hadronic model, the synchrotron emission from the high-energy proton can dominate the high-energy component in the SED of blazars (Mannheim 1993; Mücke et al. 2003). Moreover, the high-energy protons can interact with the background photons to produce the secondary electrons, the synchrotron emission from the secondary electrons can have a significant contribution to the high-energy component (Petropoulou \& Mastichiadis 2012; Mastichiadis et al. 2013; Cerruti et al. 2015; Weidinger \& Spanier 2015; Diltz \& Böttcher 2016; Zech et al. 2017). The high-energy component can also be produced by the $\pi^{0}$ decay from the protonphoton $(p \gamma)$ interactions (Sahu et al. 2013; Cao \& Wang 2014). For a recent review on blazar hadronic modelling, see Böttcher et al. (2013).

IceCube observation implied that the blazar TXS 0506+056 could be a high-energy neutrino source. The neutrinos are generally associated with the hadronic processes in the jet of blazar. High-energy neutrinos can be produced by the decay of charged pions from the $p \gamma$ interaction. Therefore, the neutrinos can be considered as the unique signature of these hadronic interactions. The recent study revealed that the multi-TeV gamma-rays of blazars can be well explained by the photohadronic process, which provides a strong evidence that the neutrino emission from blazars may originate in the photohadronic process (Sahu et al. 2019). In this paper, we study the correlated electromagnetic and neutrino emissions of blazar TXS $0506+056$ by a selfconsistent leptonic-hadronic model, taking into account both electrons and protons stochastic acceleration and all relevant radiative processes self-consistently. We reproduce the electromagnetic and neutrino emissions of blazar TXS 0506+056 by the proton synchrotron and hybrid leptonic-hadronic models based on the $p \gamma$ interaction. We demonstrate that the observed neutrino signature can allow us to distinguish these different emission models.

In Section 2 we give a brief description of the model. In Section 3 we apply the model to explain the electromagnetic and neutrino emissions of blazar TXS 0506+056. The discussion and conclusion are presented in Section 4. Throughout this paper, we adopt the cosmological parameters of $H_{0}=70$ $\mathrm{km} \mathrm{s}^{-1} \mathrm{Mpc}^{-1}, \Omega_{M}=0.3, \Omega_{\Lambda}=0.7$.

\section{2 model}

\subsection{Model geometry}

In this section, we give a brief description of the model introduced by Weidinger \& Spanier (2015). We improve the model of Weidinger \& Spanier (2015) by implementing the kinetic equation of the neutrinos and the Bethe-Heitler process. For a detail description about the model, see Weidinger \& Spanier (2010); Weidinger \& Spanier (2015).

The model assumes a spherical geometry with two zones, where a acceleration zone with radius $R_{\text {acc }}$ is nested within a larger radiation zone with a radius $R_{\text {rad }}$. Both zones are assumed to be homogeneous and to contain isotropic electron and proton distributions as well as a randomly oriented magnetic field. The considered blob travels down the jet axis towards the observer with a bulk Lorentz factor $\Gamma$, the upstream material is picked up into the acceleration zone where a highly turbulent zone is formed at the edge of the blob. Here, both injected particle species are subjected to stochastic acceleration processes up to the relativistic energies balanced by their radiation losses. However, the acceleration is assumed to be inefficient in the considerable larger radiation zone. The kinetic 
equations for each particle species $i$ in each zone can be derived from the relativistic Vlasov equation (Schlickeiser 2002) by one-dimensional diffusion approximation using the relativistic approximation $p_{i}=m_{i} c$. Since blazar jets are almost aligned with the line of sight of the observer, we assume the Doppler factor $\delta \simeq \Gamma$. All calculations are conveniently made in the rest-frame of the blob.

\subsection{Kinetic equation in the acceleration zone}

As the blob propagates through the jet, the particles from the upstream of jet are injected into the acceleration zone with an injected function

$Q_{0 . i}(\gamma)=Q_{0 . i} \delta\left(\gamma-\gamma_{0, i}\right)$

where we assume a mono-energetic and time-independent injection. Each injected particle species are continuously accelerated up to very-high energies by stochastic acceleration processes with the synchrotron and SSC losses. The energy gain due to the acceleration is balanced by the radiative and escape losses. In the acceleration zone, a hard-sphere approximation is used to describe the plasma instabilities, hence the spatial diffusion coefficient $K_{\|, i}$ is independent of particle energy (Schlickeiser 1989; Stawarz \& Petrosian 2008). This allows one to find the characteristic acceleration timescales due to stochastic acceleration processes $t_{\mathrm{acc}, \mathrm{i}}=v_{\mathrm{A}}^{2} / 4 K_{\|, i}$ (Weidinger \& Spanier 2010; Weidinger \& Spanier 2015), where $v_{\mathrm{A}}$ is Alfvén speeds. We assume the acceleration timescale to be constant and scale linearly with the particles mass. The timescale of the second species can be naturally obtained by relation $t_{\mathrm{acc}, \mathrm{i}} \propto m_{\mathrm{i}}$. Moreover, the escape timescale is set to be constant and proportional to the acceleration timescale $t_{\mathrm{esc}, \mathrm{i}} \propto t_{\mathrm{acc}, \mathrm{i}}$. We expect that the stochastic acceleration produces a power-law spectrum with spectral index $q \simeq 1+t_{\text {acc }} /\left(2 t_{\text {esc }}\right)$. This differs from the spectral index $q \simeq 1+t_{\mathrm{acc}} / t_{\mathrm{esc}}$, which is expected from diffusive shock acceleration. In fact, the stochastic acceleration produces a harder particle spectrum than the shock acceleration, which may be favorable to explain the observed SEDs from blazars (e.g., Katarzyński et al. 2006; Kakuwa et al. 2015; Diltz \& Böttcher 2016; Gao et al. 2017). In this paper, we focus on the possibility of the stochastic acceleration in blazar jets.

We assume that the acceleration zone cannot directly produce the observed SEDs, hence we only solve the kinetic equations for the primary particles. The kinetic equations for primary electrons and protons are given by (Katarzyński et al. 2006)

$$
\begin{array}{r}
\partial_{t} n_{i}=\partial_{\gamma}\left[\left(P_{s, i}(\gamma)+P_{I C, i}(\gamma)-t_{a c c, i}^{-1} \gamma\right) \cdot n_{i}\right] \\
+\partial_{\gamma}\left[\left(2 t_{a c c, i}\right)^{-1} \gamma^{2} \partial_{\gamma} n_{i}\right]+Q_{0, i}-\frac{n_{i}}{t_{e s c, i}}
\end{array}
$$

Where $P_{I C, i}(\gamma)$ is the inverse-Compton loss of each particle species, $P_{s, i}(\gamma)$ is the synchrotron loss of each particle species with

$P_{s, i}(\gamma)=\frac{4}{3} \frac{c \sigma_{\mathrm{T}}}{m_{\mathrm{e}} c^{2}} u_{B}\left(\frac{m_{e}}{m_{i}}\right)^{3} \gamma^{2}=\beta_{s, i} \gamma^{2}$,

where $\sigma_{\mathrm{T}}$ is the Thomson cross section, $u_{B}=\frac{B^{2}}{8 \pi}$ is the energy density of the magnetic field.

\subsection{Kinetic equation in the radiation zone}

As every escaping particle from the acceleration zone enters the radiation region, the particle spectrum $n_{i}(\gamma)$ from the acceleration zone severs as the injection function of the radiation zone. The particles are not accelerated in the radiation zone. Therefore, all relevant cool processes have to be taken into account, including synchrotron, inverse-Compton and photohadronic losses. The kinetic equations for electrons, protons, photons, secondary positrons and neutrinos are solved selfconsistently and time-dependently.

The low-energy photons from the primary electrons and protons are soft photons for the $p \gamma$ interactions. When the proton energy is above the threshold for the $p \gamma$ interactions, the highenergy protons will interact with the jet soft photons to produce secondary particles by the two channel:

The photon-pair prodution ( Bethe-Heitler process )

$p+\gamma \rightarrow e^{+}+e^{-}+p$.

and the photon-meson production (see, e.g., Romero \& Vila 2008; Kelner \& Aharonian 2008)

$p+\gamma \rightarrow p+a \pi^{0}+b\left(\pi^{+}+\pi^{-}\right)$,

$p+\gamma \rightarrow n+\pi^{+}+a \pi^{0}+b\left(\pi^{+}+\pi^{-}\right)$.

Where $a$ and $b$ are the pion multiplicities. The produced pions are unstable particles, they decay into stable electrons, positrons, neutrinos and $\gamma$-ray photons by the channel:

$$
\begin{aligned}
& \pi^{+} \rightarrow \mu^{+}+\nu_{\mu}, \mu^{+} \rightarrow e^{+}+\nu_{e}+\bar{\nu}_{\mu}, \\
& \pi^{-} \rightarrow \mu^{-}+\bar{\nu}_{\mu}, \mu^{-} \rightarrow e^{-}+\bar{\nu}_{e}+\nu_{\mu}, \\
& \pi^{0} \rightarrow \gamma+\gamma .
\end{aligned}
$$

This process will result in the third contribution to the VHE peak in the SEDs of blazars, besides the proton synchrotron emission and inverse-Compton emission from relativistic electrons. We use the Kelner \& Aharonian (2008) parametrization of the SOPHIA Monte Carlo results to calculate the production rate of the stable electrons, positrons, neutrinos and $\gamma$-ray photons by the interactions of equation (6). We note that the decay timescale of unstable products from the $p \gamma$ interactions is shorter compared to the synchrotron loss timescale. Therefore, we do not account for the synchrotron losses and radiation of the intermediate particles $\left(\mu^{ \pm}, \pi^{ \pm}\right)$.

The proton kinetic equation in the radiation zone is given by 


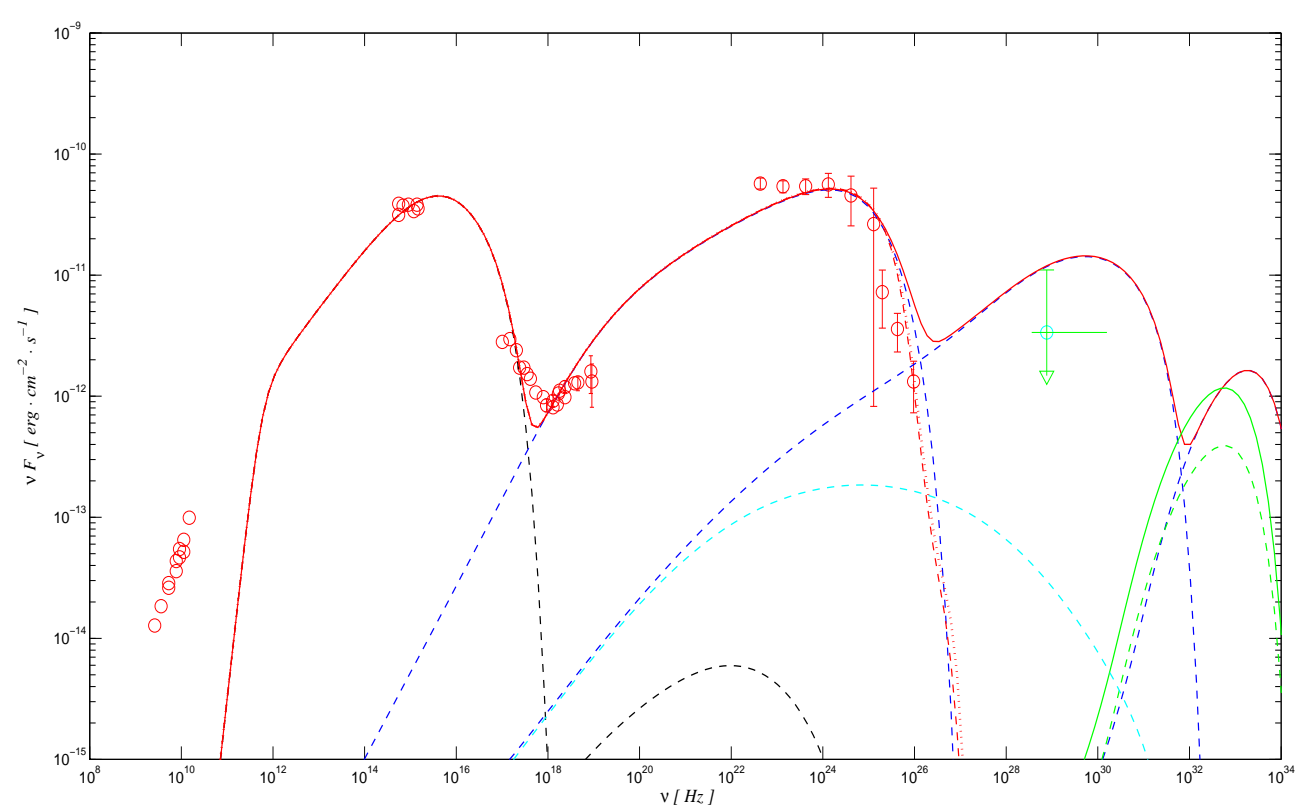

Fig. 1. Predicted multiwavelength flux and neutrino flux of TXS $0506+056$ for the proton synchrotron model. The red circles are the observed multi-wavelength data and the green circle is the detected neutrino flux from IceCube observation. The black dashed curves represent the synchrotron emission and the SSC emission, respectively (from left to right). The blue dashed curves represent the proton synchrotron emission, the synchrotron emission from the secondary pairs and the $\gamma$-ray emission from $\pi^{0}$ decay, respectively (from left to right). The cyan dashed curves represent the synchrotron emission of the secondary pairs from the Bethe-Heitler process. The green dashed curve represents the muon neutrino spectrum from the charged pions decay. All flavor neutrino spectrum is also shown as the green solid curve. The red solid curve is the total spectrum from all emission components, while the red dashed curve is the EBL-corrected spectrum using the EBL model of Finke et al. (2010). For comparison, The EBL-corrected spectrum using the EBL model of Franceschini et al. (2008) is also shown as the red dotted curve. The observed data are taken from IceCube Collaboration (2018b).

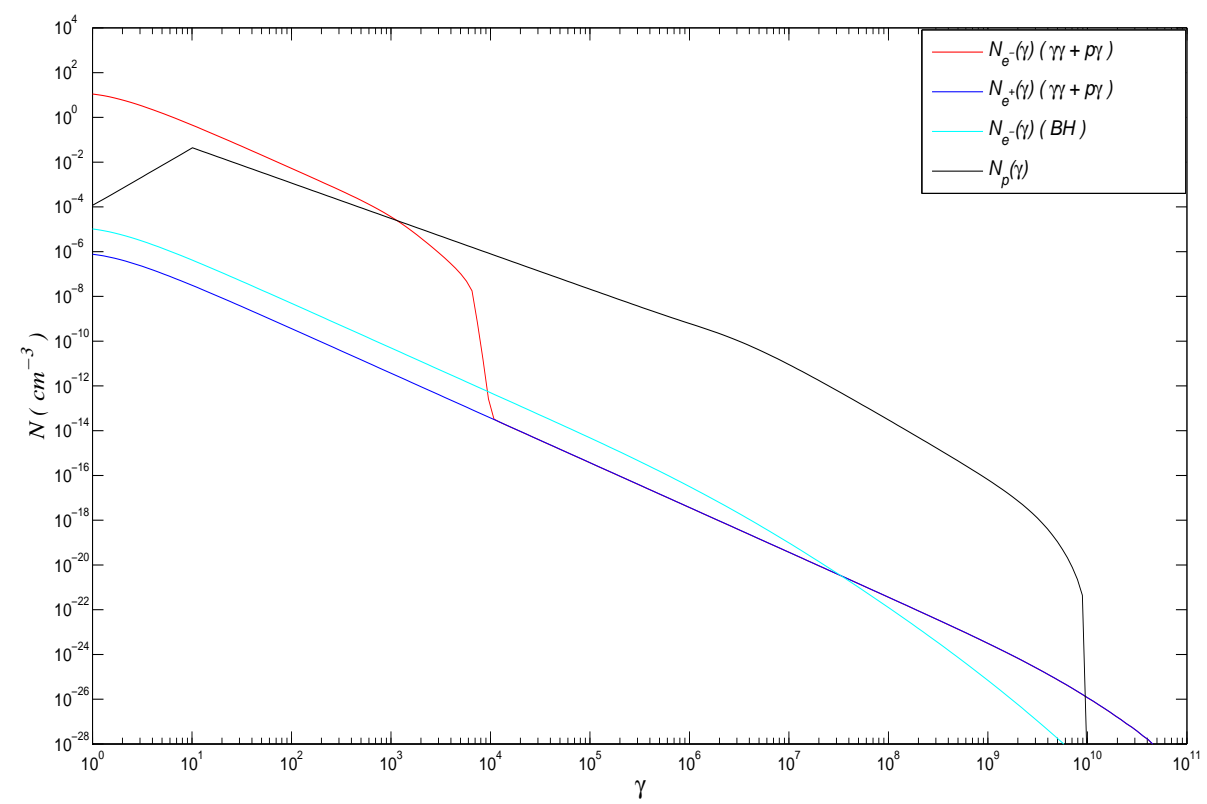

Fig. 2. Proton, electron and positron spectra from the derived SEDs in figure 1. 
Table 1. Model parameters of the proton synchrotron process

\begin{tabular}{cc}
\hline Parameters & \\
\hline$B(\mathrm{G})$ & 10 \\
$\delta_{\mathrm{D}}$ & $6.0 \times 10^{3}$ \\
$\gamma_{0, e}$ & $1.6 \times 10^{-1}$ \\
$Q_{0, e}\left(\mathrm{~cm}^{-3} \mathrm{~s}^{-1}\right)$ & 10 \\
$\gamma_{0, p}$ & $4.0 \times 10^{-3}$ \\
$Q_{0, p}\left(\mathrm{~cm}^{-3} \mathrm{~s}^{-1}\right)$ & $3.0 \times 10^{13}$ \\
$R_{\mathrm{acc}}(\mathrm{cm})$ & $1.6 \times 10^{16}$ \\
$R_{\mathrm{rad}}(\mathrm{cm})$ & 1.05 \\
$t_{\mathrm{acc}} / t_{\mathrm{esc}}$ &
\end{tabular}

$$
\begin{aligned}
\partial_{t} N_{p}= & \partial_{\gamma}\left[\left(P_{s, p}(\gamma)+P_{\mathrm{p} \gamma}(\gamma)+P_{\mathrm{BH}}(\gamma)\right) N_{p}\right] \\
& +b \frac{n_{p}}{t_{e s c, p}}-\frac{N_{p}}{t_{e s c, r a d, p}},
\end{aligned}
$$

where $b=\left(R_{\mathrm{acc}} / R_{\mathrm{rad}}\right)^{3}<1$ is a geometric factor ensuring particle conservation. We use the formula given by Kelner \& Aharonian (2008) to calculate the proton losses due to the photon-meson interaction $P_{\mathrm{p} \gamma}(\gamma)$. The proton losses from the Bethe-Heitler process $P_{\mathrm{BH}}(\gamma)$ is calculated using the formula given by Begelman et al. (1991). As in the acceleration zone, the escape timescale is assumed to scale with the particle's mass, e.g. $t_{\text {esc,rad,i }} \propto m_{\mathrm{i}}$, derived from the particle's light crossing time of a sphere with radius $R_{\text {rad }}$, multiplied by a constant empirical factor $\eta=10$.

The $\gamma$-rays from the $\pi^{0}$ decay and the secondary $e^{ \pm}$synchrotron emission are partially in the optically thick regime. The high-energy $\gamma$-ray photons can interacts with the lowenergy synchrotron photons to produce the secondary pairs by

$\gamma+\gamma \rightarrow e^{+}+e^{-}$.

This process will initiate an electromagnetic cascade until the radiation enters the optically thin regime. The secondary pairs from the $\gamma \gamma$ interactions serve as an additional injection term in the kinetic equations of electron and positron.

The kinetic equations for the electrons and positrons in the radiation zone are thus given by

$$
\begin{aligned}
& \partial_{t} N_{e^{-}}= \partial_{\gamma}\left[\left(P_{s, e}(\gamma)+P_{I C}(\gamma)\right) \cdot N_{e^{-}}\right]-\frac{N_{e^{-}}}{t_{r a d, e s c, e}} \\
&+ Q_{\gamma \gamma}(\gamma)+Q_{p \gamma^{-}}(\gamma)+Q_{\mathrm{BH}}(\gamma)+b \frac{n_{e^{-}}}{t_{e s c, e}}, \\
& \partial_{t} N_{e^{+}}=\partial_{\gamma}\left[\left(P_{s, e}(\gamma)+P_{I C}(\gamma)\right) \cdot N_{e^{+}}\right]-\frac{N_{e^{+}}}{t_{r a d, e s c, e}} \\
&+Q_{\gamma \gamma}(\gamma)+Q_{p \gamma^{+}}(\gamma)+Q_{\mathrm{BH}}(\gamma)
\end{aligned}
$$

Note that no accelerated primary positrons are assumed in the model $\left(n_{e^{+}}=0\right)$. The inverse-Compton loss rate $P_{I C}$ is calculated using the full Klein-Nishina cross section given by Blumenthal \& Gould (1970). The secondary pair production rate $Q_{p \gamma}$ from the $p \gamma$ interaction is calculated using the $\Phi_{ \pm^{-}}$ parameters of the full SOPHIA Monte Carlo calculations car- ried out by Kelner \& Aharonian (2008). The secondary pair production $Q_{\gamma \gamma}$ from the $\gamma \gamma$ interactions is calculated using the approximation of Aharonian et al. (1983). The photon-pair production rate $Q_{\mathrm{BH}}$ is calculated using the exact result of Kelner \& Aharonian (2008).

The kinetic equation for the photon field in the radiation zone is given by

$$
\begin{aligned}
\partial_{t} N_{\mathrm{ph}}= & R_{s}(\nu)+R_{c}(\nu)+R_{\pi^{0}}(\nu) \\
& -c\left(\alpha_{S S A}(\nu)+\alpha_{\gamma \gamma}(\nu)\right) N_{\mathrm{ph}}-\frac{N_{\mathrm{ph}}}{t_{\mathrm{esc}, \mathrm{ph}}},
\end{aligned}
$$

where $t_{\text {esc,ph }}=4 R_{\text {rad }} / 3 \mathrm{c}$ is the photon escape timescale. The synchrotron production rate $R_{\mathrm{S}}$ and the absorption coefficient $\alpha_{\mathrm{SSA}}$ is calculated using the exact formula given by Finke et al. (2008). The inverse-Compton production rate $R_{c}$ is calculated using the full Klein-Nishina cross section given by Blumenthal $\&$ Gould (1970). The $\gamma$-ray production rate from the $\pi^{0}$ decay, $R_{\pi^{0}}$, is calculated using $\Phi_{\gamma}$-parameters of Kelner \& Aharonian (2008). The photon annihilation coefficient $\alpha_{\gamma \gamma}$ is calculated using the exact result of Coppi \& Blandford (1990).

The neutrinos are not subject to any interaction except their production and escape. Therefore, the kinetic equation for the neutrinos are given by

$\partial_{t} N_{\nu}=R_{\nu}(\nu)-\frac{N_{\nu}}{t_{\mathrm{esc}, \mathrm{ph}}}$,

where the production rates for all favor neutrinos, $R_{\nu}(\nu)$, are calculated using the results of Kelner \& Aharonian (2008).

To model the observed SEDs of blazar, we need to transform the SEDs from the blob frame to that in the observer frame. The SEDs in the observer frame are given by

$\nu F_{\nu}^{\mathrm{obs}}\left(\nu^{\mathrm{obs}}, t^{\mathrm{obs}}\right)=\frac{h \cdot \nu^{2} \cdot N_{\mathrm{ph}}(\nu, t) \cdot \delta^{4} \cdot V_{b}}{4 \pi d_{\mathrm{L}}^{2} \cdot t_{\mathrm{esc}, \mathrm{ph}}}$

with $\nu^{\text {obs }}=\delta \nu$ and $\triangle t^{\text {obs }}=\Delta t / \delta$.

\subsection{Numeric method}

To obtain the model SEDs, we numerically solve a set of the coupled kinetic equations in the acceleration and radiation zone. In the acceleration zone, we use the method of Chang \& Copper (1970) to solve the equations (3). In the radiation zone, we use the method of Chiaberge \& Ghisellini (1999) to solve the equations (7), (9) and (10). The equations (11) and (12) are solved using the Crank-Nicolson method (Crank \& Nicotson 1996). We carefully tested our numerical code with some analytical solutions and found very good agreement.

\section{Result}

The blazar TXS $0506+056$ is a bright BL Lac objects. The redshift of the source was recently measured to be $z=0.337$ (Paiano et al. 2018). In September 2017, the IceCube reported 


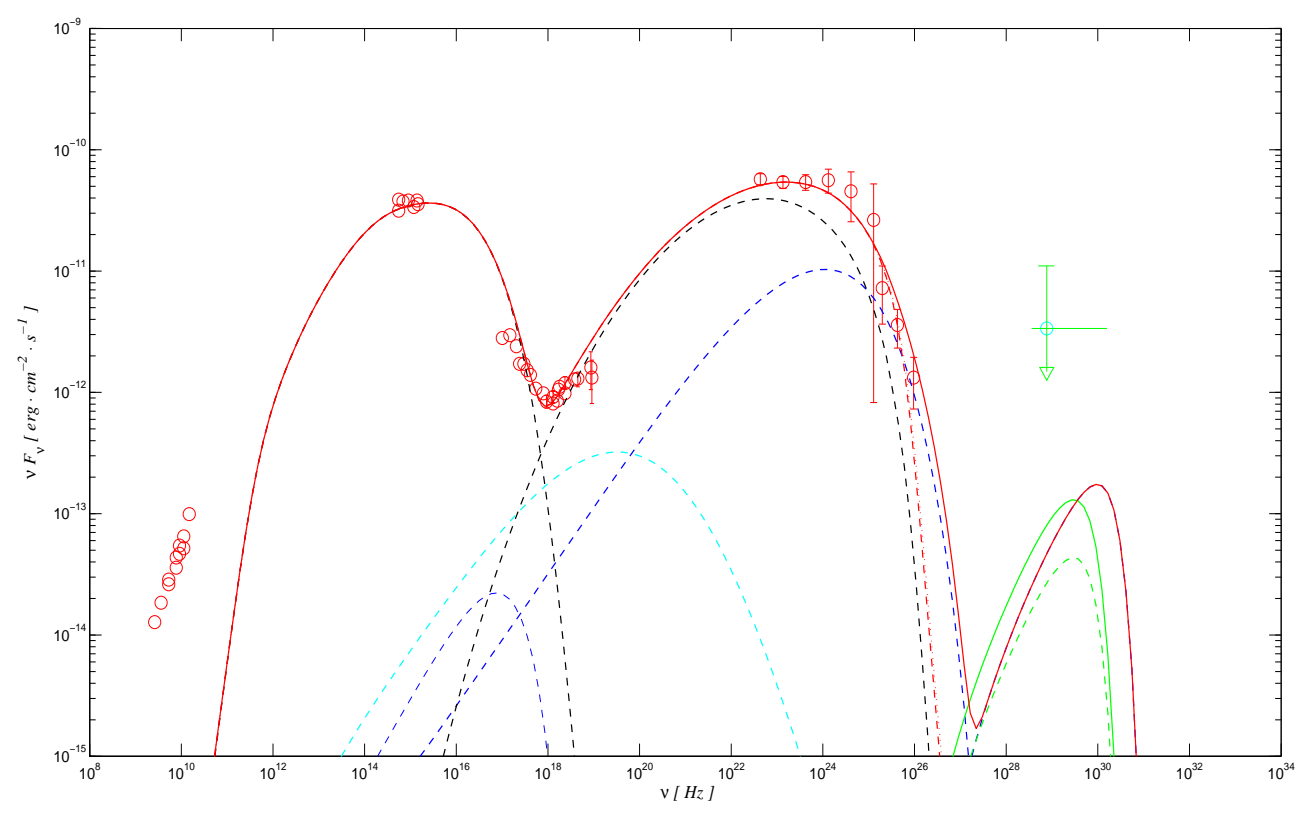

Fig. 3. Same as Figure 1, but for the hybrid leptonic-hadronic model.

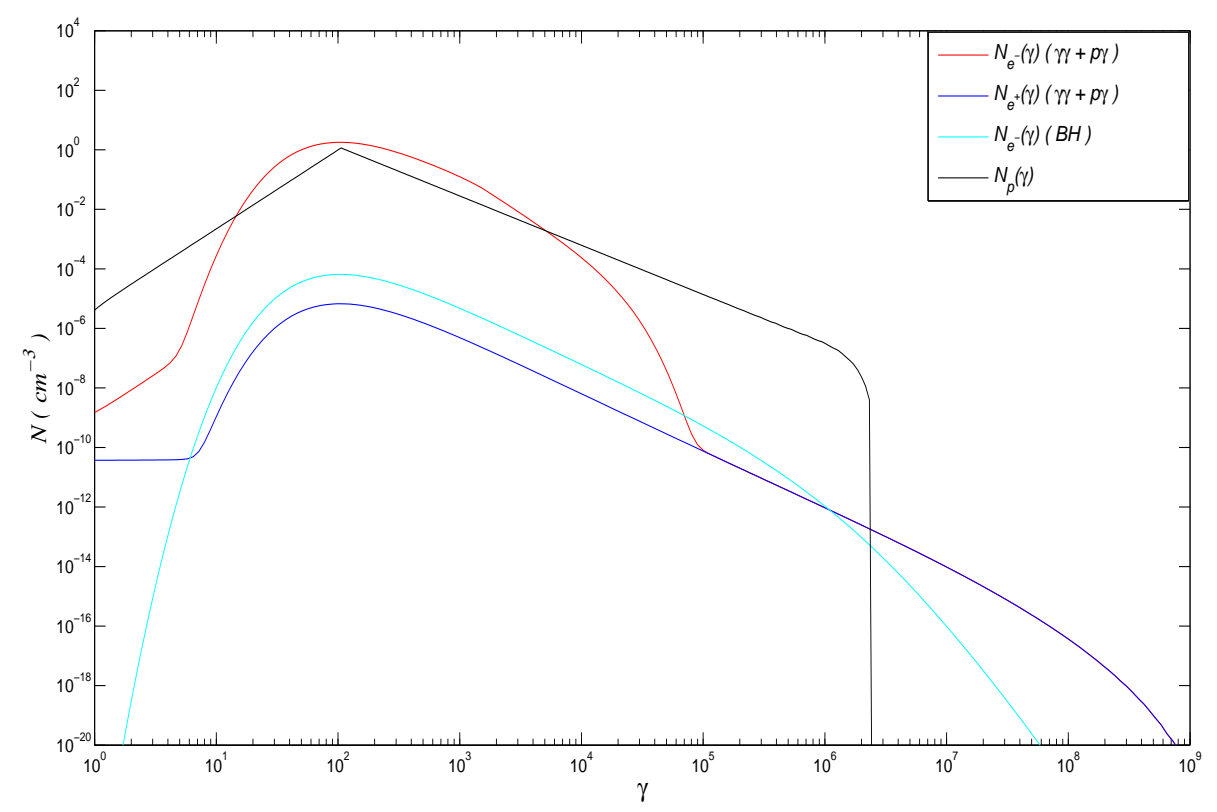

Fig. 4. Proton, electron and positron spectra from the derived SEDs in figure 3. 
Table 2. Model parameters of the leptonic-hadronic process

\begin{tabular}{cc}
\hline Parameters & \\
\hline$B(\mathrm{G})$ & 28 \\
$\delta_{\mathrm{D}}$ & $1.5 \times 10^{3}$ \\
$\gamma_{0, e}$ & $1.8 \times 10^{-1}$ \\
$Q_{0, e}\left(\mathrm{~cm}^{-3} \mathrm{~s}^{-1}\right)$ & $10^{2}$ \\
$\gamma_{0, p}$ & $1.4 \times 10^{-3}$ \\
$Q_{0, p}\left(\mathrm{~cm}^{-3} \mathrm{~s}^{-1}\right)$ & $1.0 \times 10^{14}$ \\
$R_{\mathrm{acc}}(\mathrm{cm})$ & $5.5 \times 10^{15}$ \\
$R_{\mathrm{rad}}(\mathrm{cm})$ & 1.2 \\
$t_{\mathrm{acc}} / t_{\mathrm{esc}}$ &
\end{tabular}

a very-high-energy muon neutrino event (IceCube-170922A ), which was identified by the Extremely High Energy track event selection. The best-fit reconstructed direction is $0.1^{\circ}$ from the sky position of the BL Lac object TXS 0506+056. The energy of the neutrino event is estimated to be $290 \mathrm{TeV}$ with the $90 \%$ confidence level lower limits of $183 \mathrm{TeV}$ and upper limits of 4.3 PeV, by assuming a power-law neutrino spectrum with the spectral index of -2 . The blazar TXS $0506+056$ is a $\gamma$-ray source included in the third Fermi-LAT catalog of sources (Acero et al. 2015). Following the IceCuble alert, the Fermi-LAT reported that the direction of IceCube-170922A is coincident with the location of TXS $0506+056$ and coincident with a state of the $\gamma$ ray flare (Tanaka et al. 2017). The follow-up observations were performed by a multi-wavelength campaign with different telescopes, including a significant detection by MAGIC telescopes at $>100 \mathrm{GeV}$, X-ray emissions by Swift/XRT and NuSTAR, optical emissions by the ASAS-SN survey as well as emission in radio band by VLA (see IceCube Collaboration 2018b ). The high-energy neutrino originates in the hadronic interactions providing a natural link between high-energy $\gamma$-rays and neutrino. The combined multi-wavelength and neutrino observations provide a unique opportunity to study the hadronic processes in blazar jets.

We use the model described in section 2 to model the electromagnetic and neutrino spectra of blazar TXS 0506+056. The observed data is taken from IceCube Collaboration (2018b). We interpret the electromagnetic and neutrino spectra of blazar TXS $0506+056$ by proton synchrotron and hybrid leptonichadronic models. In figure 1, we show the predicted electromagnetic and neutrino spectra for the proton synchrotron model. The modeling parameters are listed in table 1 . The derived SEDs are corrected for the EBL absorption using the model of Franceschini et al. (2008) and Finke et al. (2010). The different EBL models predict a similar $\gamma$-ray spectrum and the differences between the two EBL models are negligible. It can be seen that the optical and Swift X-ray spectra are produced by the synchrotron radiation of the primary electron, the NuSTAR X-ray spectrum comes from the low-energy tail of proton synchrotron radiation, the high-energy $\gamma$-ray spectrum is dominated by proton synchrotron radiation. In fact, the contribution of the secondary emission from the pair cascades to the $\gamma$-ray spectrum is negligible due to strong EBL absorption above $10^{26} \mathrm{~Hz}$. We note that the SSC emission from the primary electrons and the synchrotron emission of the secondary pairs from the Bethe-Heitler process have a negligible contribution to the observed SEDs due to high magnetic field of $\mathcal{O}(10 \mathrm{G}$ ) (see table 1). The peak energy of the predicted neutrino spectrum is $\sim 10^{32} \mathrm{~Hz}$, which is far above the observed neutrino energy. In figure 2, we show the proton, electron and positron spectra from the derived SEDs in figure 1. In the acceleration zone, the stochastic acceleration process produces a power-law proton injected spectrum with index $q_{p} \simeq 1+t_{\text {acc }, \mathrm{p}} /\left(2 t_{\mathrm{esc}, \mathrm{p}}\right)=1.525$. In the radiation zone, the proton synchrotron loss dominates over the photo-meson loss due to high magnetic field. Therefore, the synchrotron cooling results in a power-law proton spectrum with index $n_{p} \simeq q_{p}+1=2.525$ above the break energy $\gamma_{\mathrm{b}, \mathrm{p}} \simeq 1 /\left(t_{\mathrm{esc}, \mathrm{rad}, \mathrm{p}} \beta_{\mathrm{s}, \mathrm{p}}\right)=5 \times 10^{6}$. The maximum proton energy can be determined by $\gamma_{\max , \mathrm{p}} \simeq 1 /\left(t_{\mathrm{esc}, \mathrm{p}} \beta_{\mathrm{s}, \mathrm{p}}\right)=2 \times 10^{9}$. The peak energy of proton synchrotron radiation is $\nu_{\mathrm{s}, \mathrm{p}}^{\mathrm{obs}} \simeq$ $4.2 \times 10^{6} \delta B\left(m_{\mathrm{e}} / m_{\mathrm{p}}\right) \gamma_{\max , \mathrm{p}}^{2}=5 \times 10^{24} \mathrm{~Hz}$, which is comparable with the value from the model-derived SED (see figure 1). The electron density with $\gamma>10^{4}$ is the contribution of the secondary electrons from the pair cascade processes, because the primary electrons can not be accelerated to such high energies. We note that the X-ray and $\gamma$-ray data can not be well reproduced by the proton synchrotron model.

In figure 3, we show the predicted electromagnetic and neutrino spectra for the hybrid leptonic-hadronic model. In figure 4 , we show the proton, electron and positron spectra from the derived SEDs in figure 3. The modeling parameters are listed in table 2. It can be seen that the optical and Swift X-ray spectra come from the synchrotron radiation of the primary electron. The NuSTAR X-ray spectrum is produced by the combination of the SSC radiation and the synchrotron radiations of the secondary pairs from the Bethe-Heitler processes. The high-energy $\gamma$-ray spectrum comes from the combination of the SSC radiation and the synchrotron radiation from the secondary pairs. We note that the synchrotron radiation from the secondary pairs mainly contributes to the high-energy tails of the $\gamma$-ray spectrum. In fact, the proton synchrotron radiation makes a negligible contribution to the observed SEDs due to low magnetic field of $\mathcal{O}(1 \mathrm{G})$ (see table 2 ). The neutrino peak energy can be estimated by the maximum proton energy from the relation of $E_{\nu}=0.05 E_{\max , \mathrm{p}}$. Therefore, the corresponding neutrino peak frequency is $\nu_{\nu}^{\text {obs }} \simeq 3 \times 10^{29}\left(\gamma_{\max , \mathrm{p}} / 10^{6}\right)(\delta / 28) \mathrm{Hz}$, which is closer to the observed neutrino energy compared to the proton synchrotron emission. Recently, the study of MAGIC collaboration showed that the blazar TXS 0506+056 has a Doppler factor about 40 (Ansoldi et al. 2018). We also show the predicted 


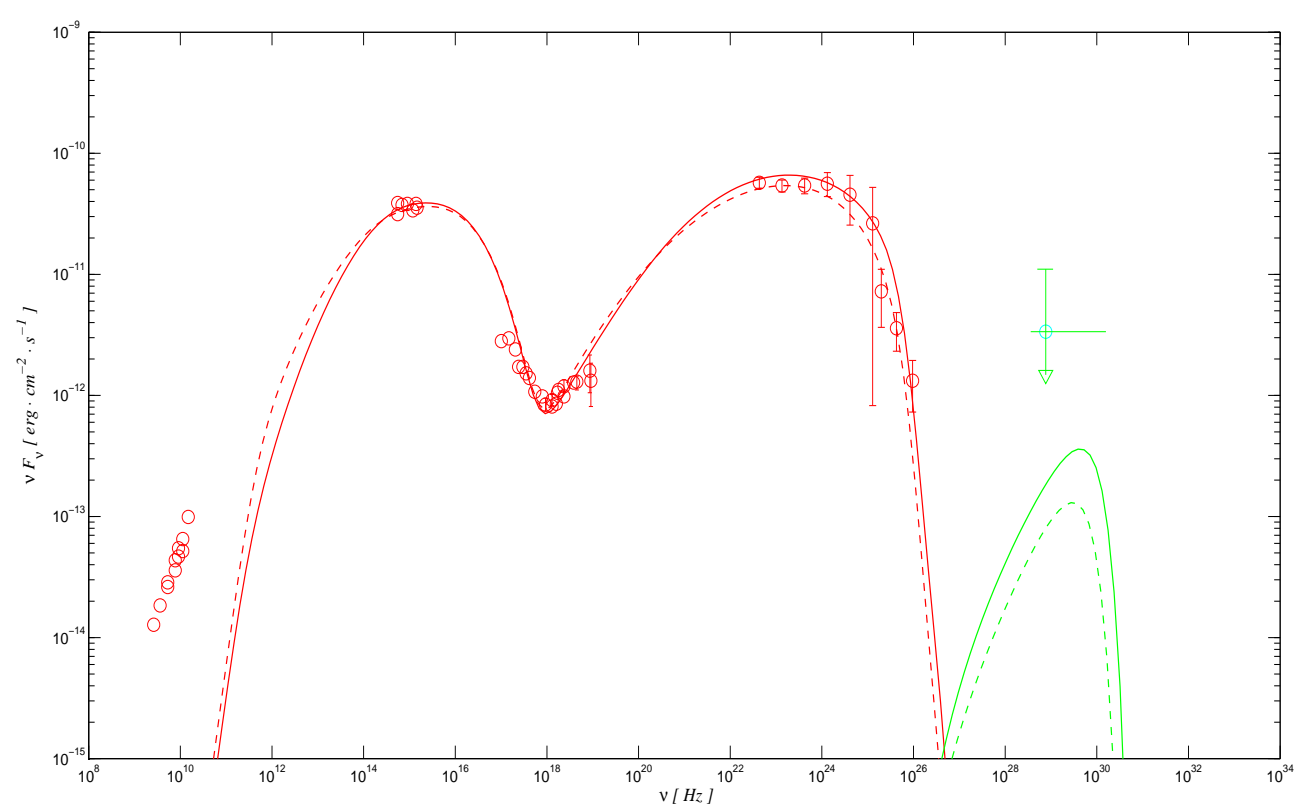

Fig. 5. Same as Figure 3, but for different Doppler factor. The solid curves represent the predicted electromagnetic and neutrino spectrum for the high Doppler factor $(\delta=40)$. For comparison, the predicted electromagnetic and neutrino spectra for the low Doppler factor $(\delta=28)$ are also shown as the dashed curves.

Table 3. Model parameters of the leptonic-hadronic process for the high Doppler factor

\begin{tabular}{cc}
\hline Parameters & \\
\hline$B(\mathrm{G})$ & 40 \\
$\delta_{\mathrm{D}}$ & $1.5 \times 10^{3}$ \\
$\gamma_{0, e}$ & $5.6 \times 10^{-2}$ \\
$Q_{0, e}\left(\mathrm{~cm}^{-3} \mathrm{~s}^{-1}\right)$ & $10^{2}$ \\
$\gamma_{0, p}$ & $2.3 \times 10^{-3}$ \\
$Q_{0, p}\left(\mathrm{~cm}^{-3} \mathrm{~s}^{-1}\right)$ & $1.0 \times 10^{14}$ \\
$R_{\mathrm{acc}}(\mathrm{cm})$ & $2.5 \times 10^{15}$ \\
$R_{\mathrm{rad}}(\mathrm{cm})$ & 1.3 \\
$t_{\mathrm{acc}} / t_{\mathrm{esc}}$ &
\end{tabular}

electromagnetic and neutrino spectra for the high Doppler factor $(\delta=40)$ as the solid curves in figure 5. The modeling parameters are listed in table 3. For comparison, the predicted electromagnetic and neutrino spectra for the low Doppler factor $(\delta=28)$ are also shown as the dashed curves. It is found that the model of the high Doppler factor predicts a slightly higher neutrino flux than one of the low Doppler factor. Our results inmpiled that the hybrid leptonic-hadronic model can better match the $\mathrm{X}$-ray and $\gamma$-ray spectra than the proton synchrotron model (see figure 1 and 5).

\section{Discussion and Conclusions}

The $p p$ interactions have been invoked to explain the observed neutrino spectrum of Blazar TXS 0506+056 (Liu et al. 2018; Wang et al. 2018; He et al. 2018; Sahakyan 2018). The $p p$ interaction usually requires the high plasma density which is not expected in the environment of BL Lac objects due to small accretion rate(Aharonian 2000). The observed neutrino spectrum can be explained by the $p \gamma$ interactions with an external radiation field (Keivani et al. 2018). It is thought that BL Lac objects are lack of a strong external radiation field due to the clean environment around the jet. A steady leptonic-hadronic model was used to study the electromagnetic and neutrino emissions of blazar TXS 0506+056(Cerruti et al. 2018; Zhang et al. 2018). However, these model do not include the particle acceleration and the relevant radiative processes self-consistently.

In this paper, we study the correlated electromagnetic and neutrino emission of blazar TXS $0506+056$ by a self-consistent leptonic-hadronic model, taking into account both electron and proton acceleration and all relevant radiative processes selfconsistently. We reproduce the electromagnetic and neutrino spectra of blazar TXS $0506+056$ by the proton synchrotron and hybrid leptonic-hadronic models based on the $p \gamma$ interaction. We find that the hybrid leptonic-hadronic model can better reproduce the observed multi-wavelength SEDs of blazar TXS 0506+056. Moreover, the predicted neutrino spectrum of the hybrid leptonic-hadronic model is closer to the observed one compared to the proton synchrotron model. Therefore, we suggest that the hybrid leptonic-hadronic model is more preferred if the neutrino IceCube-170922A originates from blazar TXS 0506+056. It is not possible to put a strong constrain on neutrino production models with single observed neutrino event. Further multi-messenger observations will be needed to understand the possible physical connection between the neutrino and $\gamma$-ray emissions. 


\section{Acknowledgments}

We thank the anonymous referee for valuable comments and suggestions. We acknowledge the financial support from the National Natural Science Foundation of China 11573060 and 11663008, the National Science Foundation of China 11673060, and the Natural Science Foundation of Yunnan Province under grant 2016FB003.

\section{References}

Acero, F., Ackermann, M., \& Ajello, M., et al. 2015, ApJs, 218, 23

Aharonian, F. A., Atoyan, A. M. \& Nagapetyan, A. M., 1983, Astrophysics 19, 187

Aharonian, F. A. 2000, NewA, 5, 377

Ansoldi, S., Antonelli, L. A., \& Arcaro, C., et al. 2018, ApJL, 863, L10

Bloom, S. D., \& Marscher, A. P. 1996, ApJ, 461, 657

Blumenthal, G. R., \& Gould, R. J. 1970, Rev. Mod. Phys., 42, 237

Begelman, M. C., Rudak, B., \& Sikora, M. 1991, ApJ, 370, 791

Böttcher, M. \& Chiang, J 2002, ApJ, 581, 127

Böttcher, M. 2007, Ap\&SS, 309, 95

Böttcher, M., Reimer, A., Sweeney, K. \& Prakash, A. 2013, ApJ, 768, 54

Blaźejowski, M., Sikora, M., Moderski, R. \& Madejski, G. M. 2000, ApJ, 545,107

Cao, G \& Wang, J. C. 2014, ApJ, 783, 108

Coppi, P. S., \& Blandford, R. D. 1990, MNRAS, 245, 453

Chang, J. S. \& Copper, G. 1970, J. Comput. Phys., 6, 1

Chiaberge, M., \& Ghisellini, G. 1999, MNRAS, 306, 551

Crank, J., \& Nicotson, P. 1996, Adv Comput Math, 6, 207

Cerruti, M., Zech A., Boisson C. Inoue S. \& Lenain, J.-P. 2018, arXiv:1807.04335

Cerruti, M., Zech A., Boisson C. \& Inoue S., 2015, MNRAS, 448, 910

Dermer, C. D., \& Schlickeiser, R. 1993, ApJ, 416, 458

Diltz, C., \& Böttcher, M. 2016, ApJ, 826, 54

Finke, J. D., Dermer, C. D. \& Böttcher, M. 2008, ApJ, 686, 181

Finke, J. D., Razzaque, S., \& Dermer, C. D., 2010, ApJ, 712, 238

Franceschini, A., Rodighiero, G., \& Vaccari, M. 2008, A\&A, 487, 837

Gao, S., Pohl, M., \& Winter, W. 2017, ApJ., 843, 109

Gao, S., Fedynitch, A., Winter, W., \& Pohl, M. 2019, Nature Astronomy, 3,88

He, H. N., Inoue, Y. Y, Inoue, S. S. \& Liang, Y. F. 2018, arXiv:1808.04330

IceCube Collaboration, 2018a, Science 361, 147

IceCube Collaboration, 2018b, Science 361, 1378

Kakuwa, J., Toma, K., Asano, K., Kusunose, M. \& Takahara, F. 2015, MNRAS, 449, 551

Katarzyński, K., et al. 2006, A\&A, 453, 47

Kelner, S. R., \& Aharonian, F. A. 2008, Phys. Rev. D, 78, 034013

Keivani, A., Murase, K., Petropoulou, M., Fox, D., et al. 2018, ApJ, 864, 84

Lin, Y. C., Bertsch, D. L., Dingus, B. L., et al. 1996, A\&AS, 120, 499

Liu, R. Y., Wang, K., Xue, R., Taylor, A. M., Wang, X. Y, Li, Z. \& Yan, H. R. 2018, arXiv:1807.05113

Maraschi L., Celotti A., Ghisellini G. 1992, ApJ, 397, L5

Mannheim, K. 1993, A\&A, 269, 67

Mastichiadis, A., Petropoulou, M., \& Dimitrakoudis, S. 2013, MNRAS, 434, 2684

Mücke, A., Protheroe, R. J., Engel, R., Rachen, J. P., \& Stanev, T. 2003, $\mathrm{APh}, 18,593$

Murase, K., Ioka, K., Nagataki, S., \& Nakamura, T. 2006, ApJ, 651, L5
Murase, K., Thompson, T. A., Lacki, B. C., \& Beacom, J. F. 2011, Phys.Rev., D84, 043003

Murase, K., Inoue, Y., \& Dermer, C. D. 2014, PhRvD, 90, 023007

Murase, K., Oikonomou, F., \& Petropoulou, M. 2018, ApJ, 865, 124

Padovani, P., Resconi, E., Giommi, P., Arsioli, B., \& Chang, Y. L. 2016, MNRAS, 457,3582

Paiano S., Falomo R., Treves A. \& Scarpa R., 2018, ApJ, 854, L32

Petropoulou M. \& Mastichiadis A., 2012, MNRAS, 421, 2325

Petropoulou, M., Giannios, D., \& Dimitrakoudis, S. 2014, MNRAS. Soc., 445,570

Petropoulou, M., Dimitrakoudis, S., Padovani, P., Mastichiadis, A., \& Resconi, E. 2015, MNRAS. Soc., 448, 2412

Petropoulou, M., Nalewajko, K., Hayashida, M., \& Mastichiadis, A. 2017, MNRAS , 467, L16

Romero, G. E., \& Vila, G. E. 2008, A\&A, 485, 623

Schlickeiser, R. 2002, Astron. Astrophys. Lib. (Berlin: Springer)

Sikora, M., Begelman, M. C., \& Rees, M. J 1994, ApJ, 421, 153

Sahu, S., Oliveros, A. F. O., \& Sanabria, J. C. 2013, Phys. Rev. D, 87, 103015

Sahu, S., López, F., Carlos, E., \& Nagataki, S. ApJL, 884, L17

Schlickeiser, R., 1989, ApJ, 336, 243

Stawarz, Ł \& Petrosian, V,. 2008, ApJ, 681, 1725

Sahakyan, N. 2018, arXiv:1808.05651

Tanaka, Y. T., Buson, S., \& Kocevski, D. 2017, ATel, 10791, 1

Wang, K., Liu, R. Y, Li, Z., Wang, X. Y. \& Dai, Z. G. 2018, arXiv: 1809.00601

Weidinger M. \& Spanier F., 2010, A\&A, 515, 18

Weidinger M. \& Spanier F., 2015, A\&A, 573, 7

Zhang, J., Liang, E. -W., Zhang, S. -N., \& Bai, J. M. 2012, ApJ, 752, 157

Zhang, H. C., Fang, K. \& Li, H. 2018, arXiv:1807.11069

Zech A., Cerruti M., \& Mazin D., 2017, A\&A, 602, A25 